\title{
The Response to Fish Oil in Patients with Heart Disease Depends on the Predominant Arrhythmia Mechanism
}

\author{
Hester M. Den Ruijter • Ruben Coronel
}

Published online: 25 April 2009

C The Author(s) 2009. This article is published with open access at Springerlink.com

Dear sir,

We have read with interest the paper by Nodari et al [1] and the editorial by Dr. Raitt [2] on fish oil fatty acids and arrhythmic risk in heart failure patients caused by idiopathic dilated cardiomyopathy. In both publications, the mechanism by which fish oils may be both pro- and antiarrhythmic are mentioned but remain un-clarified. The heterogeneous response to fish oil in patients with heart disease may be explained by the arrhythmia mechanism.

The GISSI Trial showed that long-term supplementation with fish oil reduced cardiovascular mortality following a myocardial infarction [3]. This could be attributed to a $45 \%$ reduction in sudden death, suggesting a direct antiarrhythmic effect of fish oil in these patients [4]. Subsequently, the effect of fish oil on arrhythmias was studied in patients with implanted cardiac defibrillators that suffered from a variety of cardiac pathologies [5-7]. The results were controversial; proarrhythmic and antiarrhythmic effects as well as absence of effects were documented. The recent meta-analysis by Jenkins and co-workers [8] confirmed heterogeneous outcomes in fish oil trials and concludes that fish oils need to be prescribed with caution. The question remains: Why is the response to fish oil heterogeneous? One possibility is that the diverse patient populations have different arrhythmogenic substrates (heart failure, acute ischemia, infarcted myocardium).

Circulating and membrane-bound fish oils shorten the cardiac action potential duration by modulating several ion

H. M. Den Ruijter • R. Coronel $(\bowtie)$

Experimental Cardiology Group, Heart Failure Research Center, University of Amsterdam,

Amsterdam, The Netherlands

e-mail: r.coronel@amc.uva.nl channels (for review see [9]). Shortening of the action potential, as seen in fish oil fed pigs [10], is proarrhythmic during acute myocardial ischemia [11]. Blockade of the cardiac sodium channel by fish oil may slow conduction, and favor reentrant activation. In contrast, the cardiac action potential is prolonged during heart failure, leading to triggered arrhythmias [12]. Action potential or repolarization time shortening in this setting is antiarrhythmic. In the patients with idiopathic dilated cardiomyopathy that were studied by Nodari and coworkers [1], the predominant arrhythmia mechanism likely is triggered activity. These triggered arrhythmias are inhibited by fish oil in isolated myocytes of patients with end-stage heart failure as a result of cardiac action potential shortening in combination with lower diastolic calcium levels and a reduced response to $\beta$ adrenergic stimulation [13].

Fish oils also affect heart rate, lipoprotein levels, inflammatory pathways and hypertrophy and thus also indirectly may determine whether the heart develops arrhythmia.

Altogether this leads to a complex situation; fish oil is antiarrhythmic under conditions of heart failure, and proarrhythmic under condition of acute myocardial ischemia. Patients, especially those with heart disease, may have both types of arrhythmia mechanisms. This makes the outcome of dietary supplementation with fish oil difficult to predict.

The paper of Nodari [1] nicely demonstrates that the effect of fish oil can be best studied in a well defined patient population. We are of the opinion that fish oil supplementation can be safely administered in these patients with idiopathic dilated cardiomyopathy. Whether or not this is the case for other heart disease patients depends on the main arrhythmia mechanism. 
Open Access This article is distributed under the terms of the Creative Commons Attribution Noncommercial License which permits any noncommercial use, distribution, and reproduction in any medium, provided the original author(s) and source are credited.

\section{References}

1. Nodari S, Metra M, Milesi G, Manerba A, Cesana BM, Gheorghiade $\mathrm{M}$, et al. The role of $n-3$ PUFAs in preventing the arrhythmic risk in patients with idiopathic dilated cardiomyopathy. Cardiovasc Drugs Ther. 2009;23:5-15.

2. Raitt MH. Are $n-3$ polyunsaturated fatty acids antiarrhythmic in the absence of ischemia? Editorial to: "The role of $n-3$ PUFAs in preventing the arrhythmic risk in patients with idiopathic dilated cardiomyopathy" by S. Nodari et al. Cardiovasc Drugs Ther. 2009;23:1-3.

3. GISSI-Prevenzione Investigators. Dietary supplementation with n3 polyunsaturated fatty acids and vitamin E after myocardial infarction: results of the GISSI-Prevenzione trial. Lancet. 1999;354:447-55.

4. Marchioli R, Barzi F, Bomba E, Chieffo C, Di Gregorio D, Di Mascio R, et al. Early protection against sudden death by $n-3$ polyunsaturated fatty acids after myocardial infarction: Timecourse analysis of the results of the Gruppo Italiano per lo Studio della Sopravvivenza nell'Infarto Miocardico (GISSI)Prevenzione. Circulation. 2002;105:1897-903.

5. Brouwer IA, Zock PL, Camm AJ, Bocker D, Hauer RNW, Wever EFD, et al. Effect of fish oil on ventricular tachyarrhythmia and death in patients with implantable cardioverter defibrillators: The study on omega-3 fatty acids and ventricular arrhythmia (SOFA) randomized trial. JAMA. 2006;295:2613-9.

6. Leaf A, Albert CM, Josephson M, Steinhaus D, Kluger J, Kang JX, et al. Prevention of fatal arrhythmias in high-risk subjects by fish oil $n-3$ fatty acid intake. Circulation. 2005;112:2762-8.

7. Raitt MH, Connor WE, Morris C, Kron J, Halperin B, Chugh SS, et al. Fish oil supplementation and risk of ventricular tachycardia and ventricular fibrillation in patients with implantable defibrillators: a randomized controlled trial. JAMA. 2005;293:2884-91.

8. Jenkins DJA, Josse AR, Beyene J, Dorian P, Burr ML, LaBelle R, et al. Fish-oil supplementation in patients with implantable cardioverter defibrillators: a meta-analysis. CMAJ. 2008;178:157-64.

9. Den Ruijter HM, Berecki G, Opthof T, Verkerk AO, Zock PL, Coronel R. Pro- and antiarrhythmic properties of a diet rich in fish oil. Cardiovasc Res. 2007;73:316-25.

10. Verkerk AO, van Ginneken ACG, Berecki G, den Ruijter HM, Schumacher CA, Veldkamp MW, et al. Incorporated sarcolemmal fish oil fatty acids shorten pig ventricular action potentials. Cardiovasc Res. 2006;70:509-20.

11. Coronel R, Wilms-Schopman FJG, den Ruijter HM, Belterman $\mathrm{CN}$, Schumacher CA, Opthof T, et al. Dietary $n-3$ fatty acids promote arrhythmias during acute regional myocardial ischemia in isolated pig hearts. Cardiovasc Res. 2007;73:386-94.

12. Janse MJ. Electrophysiological changes in heart failure and their relationship to arrhythmogenesis. Cardiovasc Res. 2004;61:208-17.

13. Den Ruijter HM, Berecki G, Verkerk AO, Bakker D, Baartscheer A, Schumacher CA, et al. Acute administration of fish oil inhibits triggered activity in isolated myocytes from rabbits and patients with heart failure. Circulation. 2008;117:536-44. 